this is that the mucosal environment of female reproductive tract - in the case of male-to-female transmission - is generally capable of repelling the virus. Several lines of evidence have linked HIV transmission co-factors with altered mucosal immune parameters. Mucosal inflammation, defined by elevated cytokine concentrations in cervicovaginal secretions, was associated with increased rates of HIV acquisition in the CAPRISA 004 study. While the causes of these remain unclear, we have also shown that mucosal cytokines are associated with an altered mucosal proteome including impaired barrier function, and increased frequencies of HIV target cells in the mucosa. A further proteomic analysis of HIV cases and controls demonstrated that HIV outcome could be predicted with $97 \%$ accuracy on the basis of 10 key proteins. Proteins overrepresented in cases included those associated with inflammation, while barrier-associated proteins were overrepresented in controls. Application of this model to younger women, who in the sub-Saharan African epidemic represent the group with the highest incidence rates, will be important to understanding HIV risk. In particular, the role of sex work-related exposures including rates of condom use, partner change, vaginal practices, microbiome, and other infections in driving immune changes remains poorly described. A better understanding of HIV transmission at a mucosal level may reveal novel HIV prevention options.

\section{S14.3 IMMUNE ACTIVATION, GENE EXPRESSION AND HIV ACQUISITION RISK}

${ }^{1,2,3} \mathrm{KR}^{1,2}$ Fowke ${ }^{*},{ }^{1} \mathrm{G}$ Boily-Larouche, ${ }^{2,4} \mathrm{~L}$ Mwangi, ${ }^{4} \mathrm{~J}$ Mungai, ${ }^{4} \mathrm{M}$ Akolo, ${ }^{4} \mathrm{M}$ Kimani, 1,2,3 Jyugi, 1,2,3,4 Kimani, ${ }^{1} \mathrm{~J}$ Lajoie. 'Department of Medical Microbiology, University of Manitoba; ${ }^{2}$ Department Medical Microbiology, University of Nairobi; ${ }^{3}$ Deparment of Community Health Science, University of Manitoba; ${ }^{4}$ Kenyan AIDS Control Program

\subsection{6/sextrans-2015-052270.67}

Background Sexual transmission of HIV is actually fairly inefficient and when it does occur usually a single virus is responsible for establishing the infection. Since activated HIV target cells $(\mathrm{CD} 4+\mathrm{CCR} 5+)$ are known to be $1000 \mathrm{x}$ more susceptible to HIV infection than quiescent cells, one of the known risk factors for HIV acquisition is elevated baseline levels of immune activation. This study was designed to determine if oral administration of low doses of anti-inflammatory agents, acetylsalicylic acid (ASA) and hydroxychloroquine (HCQ), would reduce the number of HIV target cells in the female genital tract.

Methods 80 low-risk women from Nairobi, Kenya established baseline immune activation levels and then were randomized to 6 weeks oral administration of low doses of either ASA $(81 \mathrm{mg} /$ day) or HCQ (200 mg/day). Cellular activation (CD69, HLADR, CD95, CCR5) was assessed by flow cytometry of peripheral blood mononuclear cells (PBMC) and cervical mononuclear cells (CMC).

Results In PBMCs, a reduction in the percentage of CD4+CD69 $+(\mathrm{p}=0.01)$ and CD4+CCR5+ $(\mathrm{p}=0.03) \mathrm{T}$ cells was observed in the ASA arm and a significant decrease of CD95 $(\mathrm{p}<0.0001)$ and CCR5 $(\mathrm{p}=0.01)$ expression on CD4+ T cells was observed following HCQ treatment. At the mucosal level, CMCs showed reduced levels of CD4+CCR5 + T cells following ASA treatment ( $p-0.02$ ) and lower expression of the activation marker CD69 on CD4+ cells in the HCQ arm ( $p=0.05)$.

Conclusions Reducing the number of HIV target cells in the female genital tract, represents a new approach to reducing HIV risk. This study showed that daily administration of low-dose anti-inflammatory drugs reduces the number of HIV target cells in the female genital tract. Further studies are required to determine if a similar reduction in mucosal HIV target cells can be observed in women at high-risk of acquiring HIV and if those reduction provide any protective benefit.

\section{S15 - Multipurpose Technologies (MPTs): developing interventions to simultaneously prevent STIs, HIV and pregnancy}

\section{S15.2 GLOBAL MAPPING OF STI, HIV AND UNPLANNED PREGNANCY: WHERE DO THESE EPIDEMICS INTERSECT?}

Erin Schelar*. United States Agency for International Development, Washington DC, USA

\subsection{6/sextrans-2015-052270.68}

Worldwide, women face sexual and reproductive health (SRH) risks including unintended pregnancy and sexually transmitted infections (STIs) including HIV. Multipurpose prevention technologies (MPTs) combine protection against two or more SRH risks into one product. Male and female condoms are the only currently available MPT products, but several other forms of MPTs are in development. We conducted a comprehensive analysis to examine the global distribution of selected SRH issues to determine where various risks have the greatest geographical overlap.

We examined four indicators relevant to MPTs in development: HIV prevalence, herpes simplex virus type 2 (HSV-2) prevalence, human papillomavirus (HPV) prevalence, and the proportion of women with unmet need for modern contraception. Using ArcGIS Desktop, we mapped these indicators individually and in combination on chloropleth and graduated symbol maps. We conducted a principal components analysis to reduce data and enable visual mapping of all four indicators on one graphic to identify overlap. Our findings document the greatest overlapping risks in sub-Saharan Africa, and we specify countries in greatest need by specific MPT indication. These results can inform strategic planning for MPT introduction, market segmentation, and demand generation, but data limitations also highlight the need for improved (non-HIV) STI surveillance globally.

\section{S15.3 MAKING THE CASE FOR MPTS: PREVENTIONS OF INFERTILITY AND OTHER STI SEQUELAE}

1,20keoma Mmeje, ${ }^{3}$ Sheryl van der Poel. 'Department of Obstetrics and Gynecology, University of Michigan Medical School; ${ }^{2}$ Department of Health Behavior and Health Education, University of Michigan School of Public Health; ${ }^{3}$ Department of Reproductive Health and Research, World Health Organization, Geneva, Switzerland

\subsection{6/sextrans-2015-052270.69}

In regions of sub-Saharan Africa where HIV is highly prevalent, HIV-affected couples require multipurpose prevention technologies (MPTs) to enhance their reproductive healthcare options beyond contraception and prevention of HIV and sexually transmitted infections (STIs). HIV-affected couples are living longer, healthier lives and are requesting options that will include assistance in becoming pregnant and establishing a family. These couples face unique challenges that require access to specialized information and reproductive services to prevent STIs or HIV transmission while attempting pregnancy. The male condom is the oldest and least expensive available MPT that effectively 
prevents HIV, STIs, and other STI-related sequelae, including infertility. Furthermore, when couples attempt pregnancy, the unique characteristics of the condom allow it to be used in conjunction with safer conception interventions during the fertile period of the menstrual cycle. The condom may be used with the following: timed intercourse and self-vaginal insemination with or without pre-exposure prophylaxis. Therefore, HIVaffected couples with an immediate desire for a child may use the condom as an MPT, and during the fertile period, the couples may choose either a clinical or pharmacological method in their attempts to achieve pregnancy. Innovative MPTs need to combine methods and technologies in order to assist HIVaffected couples in maintaining their reproductive health, to prevent HIV/STI transmission, and to achieve their reproductive goals of having healthy children when desired. The traditional view of the condom serving only as dual prevention of pregnancy and HIV/STIs needs to be reconsidered, and instead expanded to include its ability to address all disease prevention and reproductive needs of HIV-affected couples. Future designs for MPT methodologies that do encompass the multiple aspects of HIV/STI prevention and reproductive needs may impact MPT demand, uptake, acceptability, and adherence.

\section{S15.4 THE UNMET NEED AND POTENTIAL APPLICATION OF MPTS FOR RECTAL USE: IMPLICATIONS FOR WOMEN AND MSM AT RISK OF STIS AND HIV}

1,2Bridget Haire*. ${ }^{1}$ The Kirby Institute; ${ }^{2}$ Australian Federation of AIDS Organisations

10.1136/sextrans-2015-052270.70

Introduction Both men and women and transgendered people practice receptive anal sex, and an act of anal intercourse is 1020 times more likely to transmit HIV than an act of vaginal intercourse. Condoms are effective at preventing HIV acquisition through receptive anal sex, but many people do not use them perfectly for every sex act. Oral HIV pre-exposure prophylaxis is also effective at preventing HIV acquisition through receptive anal sex, but it does not prevent other sexually transmissible infections. Hence there is a need to develop a wider range of tools so that there are options for optimal protection of sexual health for men and women practicing receptive anal intercourse. Methods This paper reviews the pipeline of products in development that are designed to protect the sexual health of people practicing receptive anal intercourse.

Results A range of products are in development which are designed the rectal use. These products are 'multipurpose' technologies primarily as they aim to combine products frequently used prior to or during anal intercourse for ease and pleasure such as sexual lubricants, and douche and enema products - for HIV and potentially sexually transmissible infection (STI) prevention purposes. Other products include suppositories and specifically designed applicators for rectal use. Currently research is focused on antiretroviral-based HIV prevention technologies, but there has also been some research on non-antiretroviral-based products.In addition, there are developments in oral pre-exposure prophylaxis for STIs other than HIV.

Conclusion Given that adherence to interventions is critical to the current HIV prevention tools, building preventative agents into products that people choose to use to enhance the experience of sex may help to optimize adherence.

Disclosure of interest statement No interests to disclose.

\section{S16 - STI and HIV in pregnancy}

\section{S16.1 ELIMINATING MOTHER TO CHILD TRANSMISSION OF HIV AND SYPHILIS}

Nathalie Broutet* World Health Organization, Geneva, Switzerland

\subsection{6/sextrans-2015-052270.71}

The Initiative for Global Elimination of Congenital Syphilis was launched in 2007, and the Global Plan towards the Elimination of New HIV Infections among Children by 2015 and Keeping Their Mothers Alive was launched in 2010. In 2014, the World Health Organization, jointly with UNAIDS, UNICEF, UNFPA, and other global partners released guidance on the processes and criteria for dual elimination of mother-to-child transmission (MTCT) of HIV and syphilis. Countries all over the world are now striving for dual elimination. This presentation will address recent successes like Cuba and current strategies and new tools that can help to overcome barriers in the dual elimination of MTCT HIV and syphilis with a particular focus on MTCT of syphilis.

\section{S16.2 POTENTIAL FOR UPTAKE OF DIAGNOSTIC TESTING SERVICES ALONG THE CONTINUUM OF CARE: LANDSCAPE ASSESSMENT OF COMMUNITY AND PROVIDERS}

Sadaf Khan*, Matt Steele, Lynn Atuyambe, Peter Waiswa, Nabeel Ashraf Ali, Dunia Faulx, Shamsul Arifeen. PATH, Seattle, USA

\subsection{6/sextrans-2015-052270.72}

Introduction Maternal infections continue to be a major contributor to maternal, perinatal, and neonatal morbidity and mortality. The use of diagnostic technologies for maternal infections in the developing world is poorly researched. The current study aimed to elucidate the provider- and patient-level factors that may act to inhibit or encourage timely and effective provision of diagnostic technologies during the pregnancy, intrapartum, and early postpartum periods for infections linked with poor outcomes for mothers and infants in the developing world.

Methodology This was an exploratory study that employed a socio-ecological framework. Using qualitative data collection methods, including focus group discussions (FGDs) with pregnant women, new mothers and their families, and in-depth interviews (IDIs) with lower-level providers, we examined healthseeking behaviours and perceptions of diagnostic testing during pregnancy with the aim of obtaining an in-depth understanding of factors related to the potential for uptake of new diagnostic technologies in two developing country settings (Bangladesh and Uganda).

Results Diagnostic testing during ANC was generally viewed positively. Findings suggest that women and their families would prefer any proposed diagnostic tests be administered by skilled and trained providers. Communities expressed reservations in providing biological specimens during labour and the immediate postpartum period with discomfort and pain during labour and postpartum confinement cited as reasons for these preferences. Blood and urine were deemed the most acceptable specimens, and vaginal swabs were generally not viewed favourably. While providers were familiar with the concept of diagnostic testing, there is limited use of diagnostics in maternal health care, with HIV testing in Uganda being a notable exception. While 